\title{
Análisis de la implicación de la diversidad de actores en el Plan Integral del Polígono Sur: una mirada sistémica e integrada
}

\author{
Analysis of the involvement of the diversity of actors \\ in the Comprehensive Neighborhood Plan of the \\ Poligono Sur: a systemic and integrated approach
}

\author{
Cristina Gallego Gamazo*
}

Fecha de recepción: 04-05-2016 - Fecha de aceptación: 23-09-2016

Hábitat y Sociedad (ISSN 2173-125X), n. ${ }^{\circ}$ 9, noviembre de 2016, pp. 55-74.

\section{Summary}

In today's urban context, characterized by the manifestation of more and new urban inequalities, it is worth inquiring how the complex condition of multi-deprived urban areas is being addressed, and analyze the level of involvement of the diversity of actors from a systemic and integrated approach.

In order to face this challenge, it is proposed to advance the methods and tools for the analysis of the processes taking into consideration a multi-dimensional, multi-agent and multi-scale perspective, based on the analysis of the level and forms of participation of the diversity of actors. To that end, the transformation process driven by the Comprehensive Plan for the Polígono Sur in Seville is taken as reference. Specifically, the evaluation is done at a turning point due to the change of the management body, which is interesting to recognize the development and possible changes in this first stage to redirect the new one.

As a result, the development and application of this method focused on the logic of the relations and forms of organization, facilitates the identification of those strategic relationships enhancing the options for change, which will be of great interest to the design, monitoring and evaluation of other local development processes in multi-deprived urban areas.

\section{Key words}

Process Analysis; Participatory Research; Collaborative Planning; Situational Planning; Community Participation.

\section{Resumen}

En el contexto urbano actual, caracterizado por la manifestación de más y nuevas desigualdades urbanas, sería bueno revisar cómo se está abordando la situación compleja de los barrios más desfavorecidos y analizar la implicación de la diversidad de actores desde un enfoque sistémico e integrado.

Ante este reto, se propone avanzar métodos e instrumentos para el análisis de procesos que tengan en cuenta las múltiples dimensiones, agentes y escalas, integrando el análisis del nivel y las formas de participación de la diversidad de actores. Para ello, se toma de referencia el proceso de transformación impulsado por el Plan Integral del Polígono Sur en Sevilla. En concreto, la evaluación se realiza en un momento de inflexión debido al cambio en la dirección del ente de gestión, que permite reconocer el desarrollo y los posibles cambios a lo largo de la primera fase y reorientar la nueva.

Como resultado, el desarrollo y aplicación de este método centrado en la lógica de las relaciones y formas de organización facilita la identificación de aquellas relaciones estratégicas que potencian las opciones de cambio, que será de gran interés para el diseño, seguimiento y evaluación de nuevos procesos de desarrollo local de áreas urbanas desfavorecidas.

\section{Palabras clave}

Análisis de Procesos, Investigación Participativa, Planeamiento Colaborativo, Planificación Estratégica Situacional, Participación Ciudadana

\footnotetext{
* Grupo de investigación ABIO: Arquitectura Bioclimática en un entorno sostenible. Escuela Técnica Superior de Arquitectura, Universidad Politécnica de Madrid. Avenida Juan de Herrera 4, CP 28040, Madrid. E-mail: c3gc3g@gmail.com
} 


\section{Introducción: la importancia de la participación para actuar de manera integrada}

En el momento actual, en el que se observa una clara separación entre los instrumentos de planeamiento y la dimensión social del espacio (Sevilla, 2010), y también la aparición de más y nuevas desigualdades urbanas, en algunas ocasiones incluso provocadas y/o intensificadas por políticas y estrategias de intervención poco acertadas (Borja, 2003; Subirats, 2004; Blanco et al., 2006; Castells, 2006), resulta importante cuestionar el papel del planeamiento urbano a la hora de atender el carácter relacional y dinámico de las situaciones de desigualdad urbana (Torres, 2003; Bonet, 2006). Esta situación requiere poner en valor la visión y la implicación de la diversidad de actores urbanos en la transformación del área como vía para cambiar la realidad de los propios afectados (Harvey, 2008).

Ante este desafío, cabe retomar la pregunta que lanzaba Jane Jacobs a principios de la década de los 60 en "Muerte y Vida de las grandes ciudades americanas" frente a las operaciones de reestructuración y remodelación profunda de los "barrios bajos" en las grandes ciudades americanas. Ante esta situación, cuestiona el papel de los poderes políticos y del mercado, y defiende la importancia de tener en cuenta los usos y las relaciones de los habitantes a la hora de hacer ciudad.

¿Cómo podemos crear ciudad de manera más efectiva trabajando con y no en contra de los patrones de uso y de relación de los habitantes promovidos por los modos de vida y el conocimiento presente en los entornos urbanos? (Jane Jacobs, 1961/2011).

Se trata de una pregunta que sigue siendo pertinente ya que advierte de la importancia de reflexionar sobre la manera en que se está abordando la problemática de los barrios más desfavorecidos, poniendo en valor la visión y la implicación de la diversidad de actores urbanos si se quiere atender las múltiples dimensiones de la situación de manera integrada.

Analizar y abordar la situación compleja de los barrios más desfavorecidos de manera integrada resulta de gran interés si tenemos en cuenta que en la actualidad:

- Se trata de un tema prioritario que responde a una preocupación generalizada en el panorama europeo y español sobre los problemas de los barrios desfavorecidos y el desarrollo sostenible del conjunto de la ciudad. Este hecho se aprecia en el enfoque estratégico de la regeneración urbana integrada que se defiende en la agenda europea y en la actualización reciente de la normativa en España, aunque se centre básicamente en las implicaciones económicas (Castrillo et al., 2014).

- Se acepta que se trata de una problemática de base estructural, relacional, multidimensional y dinámica, que requiere la acción pública y también la capacidad transformadora de la acción cívica (OCDE, 1998; Bonet, 2006; Torres, 2013).

- Se cuenta con una experiencia reciente acumulada de intervención en barrios que ha puesto en evidencia las limitaciones de los modelos tradicionales de desarrollo, planeamiento, análisis y evaluación, y al mismo tiempo, el potencial del conocimiento y de 
las condiciones y capacidades generadas en procesos participativos para el desarrollo integrado del área.

- Se están tomando diferentes caminos para hacer efectivo el Derecho a la Ciudad como alternativa frente a los efectos negativos de la globalización en las condiciones de vida urbana.

La reinterpretación actual del Derecho a la Ciudad trata la participación como un proceso creativo y transformador. Desde una mirada crítica, Harvey (2008) considera que este derecho no tiene que ver solo con el acceso individual a recursos urbanos, sino también con el derecho a participar en la transformación de la ciudad en función de nuestros deseos en un proceso que nos transformará a nosotros mismos. En esta línea, solo podrá ser entendido desde una idea revolucionaria de apropiación del derecho a la vida urbana mediante la movilización social y la lucha política y social colectiva (Marcuse, 2012; Mayer, 2012).

Si trasladamos estas ideas al mundo iberoamericano, muchos teóricos críticos defienden el derecho a la ciudad más allá del plano físico. Se considera que el hábitat es un sistema complejo de dimensiones interrelacionadas, la física o URBS, la social o CIVITAS y la política o POLIS, en un proceso que no es ajeno a posibles tensiones y conflictos (De Manuel, 2008).

Esto plantea diferentes retos. Por un lado, cuestiona la manera de introducir la producción y gestión social del hábitat en las políticas de desarrollo urbano y regional, que como señala Ortiz (2010) exige sistematizar los procesos de gestión de los organismos públicos para que sean capaces de gestionar con eficacia redes complejas de relaciones cambiantes. Sungranyes (2010) reconoce que son estrategias articuladas entre sí y que tienen lógicas de continuidad en el tiempo que propician que se empiece a tratar en marcos constitucionales y normativos.

Por otro lado, la complejidad de los sistemas de relación y de los procesos de producción, requiere formar técnicos para trabajar de manera transdisciplinar y transectorial. Para ello, Pelli (2010) propone promover espacios de aprendizaje de la gestión en los que se incorpore la formación a la gestión real.

Sin embargo, a pesar de estas evidencias y consideraciones, estos aspectos no se tienen en cuenta en los análisis y hace falta desarrollar métodos y herramientas de análisis que aborden estos procesos en toda su complejidad. Sería deseable poner en valor las capacidades previas y generadas a lo largo del proceso para una buena gestión social del hábitat.

Ante esta realidad, este trabajo centra la atención en la importancia de desarrollar análisis críticos y constructivos a lo largo del proceso de un plan de regeneración urbana. Se persigue así hacer frente a las limitaciones del procedimiento habitual en el que como mucho se realiza un diagnóstico inicial de definición de objetivos a cumplir y la evaluación final de objetivos cumplidos, lo que deja al margen la valoración de resultados intermedios y aspectos a potenciar y a corregir a lo largo del proceso, que como apuntan Gittel y Vidal (1998) son especialmente importantes para el desarrollo de comunidades desfavorecidas.

En ese sentido, el objetivo principal de este trabajo es proponer un método de análisis sistémico e integrado a partir de las lecciones del proceso impulsado por el Plan Integral del Polígono Sur, que aporte nuevas herramientas para visibilizar aspectos sobre la organización y 
coordinación de la actuación de la diversidad de actores sobre los que es necesario trabajar a lo largo del proceso.

\section{Situación de partida: grandes retos metodológicos e instrumentales}

Estudios recientes muestran que la nueva configuración social y territorial neocapitalista ha propiciado más y nuevas desigualdades urbanas (Hernández, 1997; Hernández et al., 2016; Blanco et al., 2006), de carácter estructural, relacional y dinámico (Castel, 1995, [1992]; Bonet, 2006; Vorauer, 2011; Torres, 2013), en procesos en los que entran en juego múltiples dimensiones, actores y escalas.
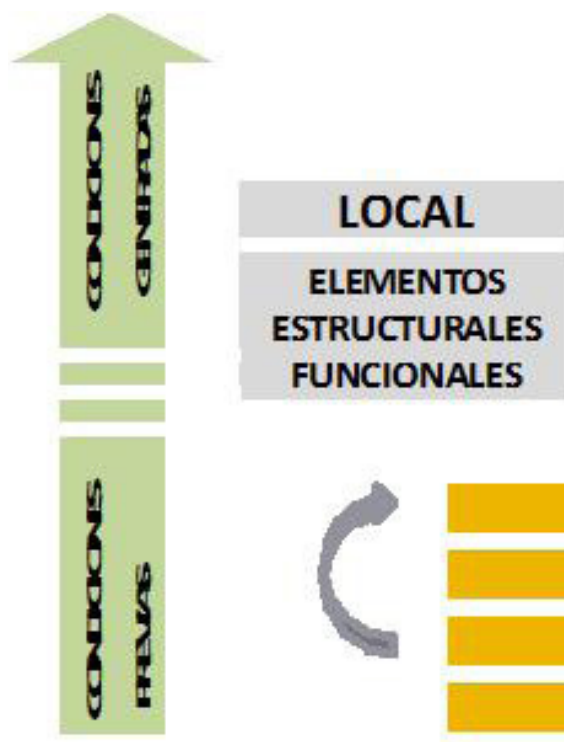

Relaciones de interdependencia

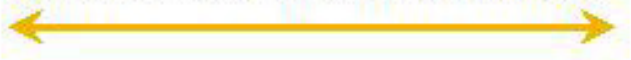

Modelo urbano territorial

Modelo de gobernanza

\section{SUPRALOCAL \\ ELEMENTOS ESTRUCTURALES FUNCIONALES}

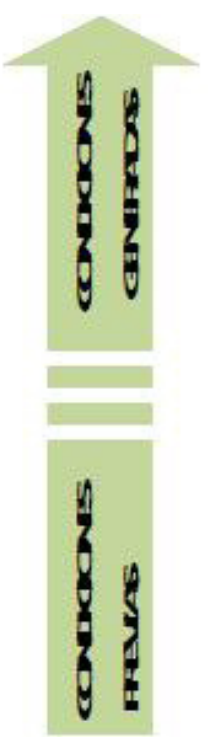

Figura 1. Esquema de la situación compleja, dinámica, multi-dimensional, multiescala y contextual de las dinámicas urbanas. Elaboración propia.

\section{Dimensión físico-espacial}

Dimensión social

Dimensión económica

Dimensión política

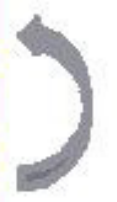

Por tanto, como se aprecia en la Figura 1, es necesaria una respuesta integrada que aborde las relaciones de interdependencia entre las dinámicas locales y supralocales en distintas dimensiones que estarán condicionadas por elementos estructurales y funcionales a nivel local y supralocal, es decir, por el modelo urbano territorial y de gobernanza, y además, por las condiciones de partida y generadas de comunicación e interacción que explican los posibles cambios en las lógicas de relación.

Así mismo, reconocer el carácter integrado del problema pone de manifiesto grandes retos. En la revisión de la práctica reciente se observa que los principales retos se deben principalmente a limitaciones administrativas y a la falta de una cultura urbana tanto entre la ciudadanía como en las instituciones (Nel.lo, 2008; Rubio, 2011).

A nivel metodológico, nos encontramos con el reto de abordar el análisis de dinámicas urbanas y procesos complejos y el análisis de políticas complejas y transversales determinadas por los flujos de relaciones y el contexto social e institucional en cada caso. De manera que es necesario desarrollar marcos y herramientas conceptuales de análisis que ayuden a reconocer el alcance social y político de este tipo de procesos, los cambios sustantivos en las formas de relación y en las condiciones y capacidades de partida, y las visiones y espacios compartidos generados gracias a la implicación activa de la población en el diagnóstico y el seguimiento. 


\section{Marco teórico y conceptual}

Se reconoce que asumir la naturaleza multi-dimensional, multi-agente y multi-escala del desarrollo local presenta un importante valor analítico y estratégico a la hora de llegar a análisis más precisos y formular políticas públicas que articulen mejor las dinámicas territoriales con los procesos sociales de aprendizaje e innovación colectiva (Fernández y Dundas, 2008).

A nivel conceptual, desde esta visión relacional y dinámica, tal y como se refleja en la Figura 2, el espacio se interpreta como una construcción social, es decir, como un espacio configurado por una serie de procesos de (re)-construcción continua de relaciones y comportamientos sociales que configuran un sistema complejo y cambiante de actores y relaciones en distintas dimensiones. La lógica de estas relaciones dependerá del contexto social e institucional en el que se da, de la trayectoria histórica, de las aspiraciones de futuro compartidas, y de los recursos relacionales disponibles para transformar esas aspiraciones en acciones colectivas (Gittel y Vidal, 1998; Moulaert, 2000; Bonet, 2006; González y Vigar, 2008).

Esta lógica relacional requiere analizar las relaciones existentes y generadas en el proceso entre actores con distintos intereses, recursos y capacidades, al igual que las debilidades y oportunidades para potenciar los recursos relacionales. Además, en la práctica, implica movilizar y coordinar la actuación de la diversidad de actores en una estrategia común.

Si además tenemos en cuenta la importancia del proceso en sí mismo, desde una postura post-positivista se entiende que el análisis de procesos deliberativos requiere ir hacia análisis cualitativos que se basen en métodos discursivos que pongan en valor aspectos intangibles difíciles de evidenciar pero fundamentales al estudiar la interacción entre agentes, y en particular, aspectos como las condiciones, las capacidades y el conocimiento generado por medio del diálogo y la acción colaborativa (Gottweis, 2003; Hajer y Wagenaar, 2003; Healey, 2003; Innes y Booher, 2003).

En este sentido, para guiar el análisis de los sistemas de relaciones en términos de capacidades, se considera la visión de Gittell y Vidal (1998) sobre el potencial del capital social como estrategia de desarrollo local. Los autores ponen la atención en los resultados intermedios de las estrategias de desarrollo local, en especial, en los resultados en cuanto el nivel de compromiso, la capacidad, el control, el capital social de vínculo (bonding) y de puente o estructura (bridging) y la fuerza de las redes (networking).

De hecho, en trabajos empíricos recientes (Forrest y Kearns, 2001; Subirats, 2004; Schnur, 2005; Skidmore, Bound y Lownsbrough, 2006; Bonet, 2006) se demuestra el papel del capital social y de las capacida-

Figura 2. Esquema de la conceptualización de la visión relacional del hábitat: multi-dimensional, multi-agente y multi-escala. PT: responsables políticos y técnicos; SE: agentes socioeconómicos; BC: base ciudadana; $\mathrm{AE}$ : asesores externos. Elaboración propia. 
Figura 3. Esquema sistémico de la acción integrada y participativa en los procesos de mejora de barrios. Elaboración propia. des institucionales que se fomentan en las redes de colaboración, que funcionan como recursos relacionales y dinámicos importantes para el desarrollo local si se asume la dimensión territorial de los problemas de cohesión social y su relación con el capital social y con el nivel de organización.

Esta visión relacional y procesual de la situación compleja de los barrios más desfavorecidos exige analizar las formas en que se relacionan actores de distintos sectores y escalas (Salet y Majoor, 2008; Blanco, Bonet y Walliser, 2011), desde una perspectiva "multi-disciplinar" y "multi-escala" (Gualini, 2006), para poder reconocer el alcance del capital social generado mediante estas relaciones (Forrest y Kearns, 2001; Skidmore, Bound y Lownsbrough, 2006).

\section{Marco metodológico}

Teniendo en cuenta estas premisas del marco teórico y conceptual, se propone establecer un método de análisis basado en la reflexión a partir de la observación y la visión de la diversidad de actores recogida en entrevistas en profundidad y grupos de discusión. Se sigue un enfoque sistémico que permita una aproximación a realidades y procesos complejos (Morín, 1990; Montañés, 2009) y un enfoque en capacidades (Sen, 1992, 2005; Healey, 1997, 1998, 2003).

Para definir este método de análisis se toma de referencia el caso del Plan Integral del Polígono Sur, con la intención de reconocer mediante la observación directa las principales formas de relación que han impulsado cambios frente a los bloqueos de partida para la regeneración integrada de la zona y también las lógicas y los cambios de estas relaciones en momentos clave del proceso.

El propósito final de esta evaluación es poner en práctica un método alternativo de análisis de estos procesos en el que se tenga en cuenta la manera en la que se han implicado la diversidad de actores a lo lar-

\section{(-) CONDICIONES EXTERNAS (+)}

SUPRALOCAL

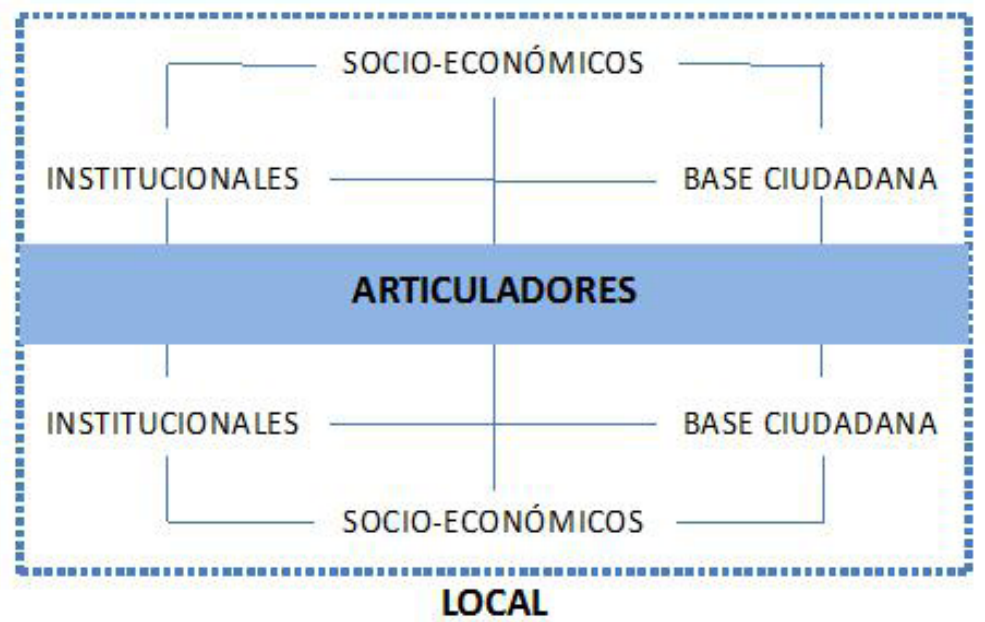

LOCAL
DINAMIZADORES GENERADOS

$(-)$ CONDICIONES INTERNAS (+) 
go del proceso y, a su vez, extraer para este caso concreto aspectos críticos a corregir y condiciones básicas que sería deseable favorecer a la hora de facilitar la conexión entre actores de diferentes sectores "en y entre" distintas escalas, y valorar de esta manera el alcance y la validez de análisis de este tipo.

Desde una perspectiva analítica conceptual, a la hora de analizar las opciones para llegar al modelo de desarrollo buscado, será necesario identificar y analizar primero los actores de diferentes sectores y escalas que pueden influir en la transformación del área, y los factores que condicionan la implicación activa y coordinada de los mismos, tal y como se refleja en la figura 3 .

Además, sería interesante completar este análisis con un estudio sobre la manera en que se han implicado la diversidad de actores a lo largo del proceso de planificación, entendido no solo como un proceso puramente instrumental, sino como un proceso estratégico de reflexión-acción-reflexión. Para ello se definen los requisitos básicos para una implicación activa y coordinada en el diagrama de momentos del proceso de planificación, en base a las recomendaciones de la planificación estratégica, integrada, participativa y situacional, y la revisión de estos requisitos en el caso del Plan Integral del Polígono Sur, siguiendo el diagrama de la Figura 4.

PROCESO DE PLANIFICACIÓN MODULAR: proceso de reflexión / acción / reflexión

\begin{tabular}{|c|c|c|c|c|}
\hline \multicolumn{1}{|c|}{$\begin{array}{c}\text { SITUACIÓN DE } \\
\text { PARTIDA } \\
\text { [SP] }\end{array}$} & $\begin{array}{c}\text { Momento } \\
\text { ANÁLISIS } \\
\text { ESTRATÉGICO } \\
\text { [AE] }\end{array}$ & $\begin{array}{c}\text { Momento } \\
\text { PROPUESTA } \\
\text { VIABLE } \\
\text { [PV] }\end{array}$ & $\begin{array}{c}\text { Momento } \\
\text { ELABORACIÓN } \\
\text { DEL PLAN } \\
\text { [EP] }\end{array}$ & $\begin{array}{c}\text { Momento } \\
\text { ACCIÓN } \\
\text { COLECTIVA }\end{array}$ \\
[AC]
\end{tabular}

\section{El Plan Integral del Polígono Sur}

\section{Contextualización del estudio}

Como se ha explicado ya, la evaluación del Plan Integral del Polígono Sur se realiza en 2013, justo en el momento de cambio de la dirección y la estructura del ente de gestión. Este análisis se utilizará para reconocer el peso de formas de relación estratégicas y de los requisitos establecidos para una implicación activa y coordinada a lo largo del proceso. Para ello se tendrán en cuenta los cambios detectados en cuanto a las formas de relación, el papel y el nivel de implicación e interacción entre actores.

Se trata de un momento en el que todavía se vive en el Polígono Sur una situación de segregación y exclusión dentro de la estructura global de la ciudad, que sería necesario abordar en las diferentes escalas de esta problemática estructural, tal y como remarca Torres tras investigar la condición de marginalidad en la ciudad de Sevilla (2013) y la situación en el caso del Polígono Sur (2005). Estudio, este último, realizado antes de acordar la elaboración del Plan Integral. En el caso particular del Polígono Sur, Torres detecta una correlación entre la concentración de situaciones de desventaja social y el grado de degradación del
Figura 4. Esquema de requisito del diagrama de momentos de la planificación. Elaboración propia. 
espacio público, de la vivienda y de la escena urbana. Por ello defiende que aunque el nivel de dotación de infraestructuras y equipamiento y de servicios públicos es aceptable respecto al conjunto de la ciudad, es necesario evaluar el papel de los mismos no solo de manera cuantitativa, sino también cualitativa. Recomienda valorar si ayudan a hacer frente a la situación de aislamiento y exclusión, o si por el contrario, la están fomentando.

Como resultado del trabajo del primer Comisionado iniciado en 2004, se mejoran las condiciones físicas de las zonas más degradadas. José M. a López (2012) recoge en su Tesis sobre "El diseño participativo en programas de rehabilitación de viviendas" las lecciones aprendidas durante su implicación en el equipo Surco que se encarga por concurso del programa de rehabilitación. Explica el difícil proceso de diálogo con los vecinos para llegar a soluciones que den respuesta a sus demandas. Esta forma de trabajar choca con el procedimiento inicial de la extinta Empresa Pública del Suelo de Andalucía (EPSA) que impuso un programa de intervención sin tratarlo previamente con los vecinos. En este proceso se han generado dinámicas de entendimiento y se ha ganado experiencia práctica en fórmulas de gestión social de la mejora de barrios, que se han visto perjudicadas por los sucesivos recortes del programa y el cambio de enfoque en el trabajo del Comisionado a una visión más técnica. La lectura de este proceso en este momento de incertidumbre resulta interesante de cara identificar oportunidades de futuro.

\section{El ámbito de actuación}

Se trata de un área de gran extensión con límites definidos por elementos naturales y urbanísticos claros que remarcan la condición de aislamiento, las vías del ferrocarril al oeste, la Carretera de Su Eminencia de tráfico rápido al sur, el frente de los polígonos industriales al este y el Parque Celestino Mutis y la Ronda Tamarguillo al norte. Engloba seis barriadas (La Oliva, Paz y Amistad, Las Letanías, Murillo, Antonio Machado y Martínez Montañés) en el extremo sur de la ciudad, con un total de 145 hectáreas y casi 9000 viviendas, más de 7000 de promoción pública.

A pesar de que en el conjunto predomina una estructura urbana de manzanas de bloque abierto que confiere cierta homogeneidad, las diferencias actuales entre las barriadas son evidentes. Se experimenta una concentración creciente de marginación social que va del norte al sur y de los límites del polígono hacia el centro (Torres, 2005). Además, esta estructura da lugar a espacios libres e interbloque de gran dimensión que remarcan aún más la separación entre sectores dentro del polígono y de este con el exterior.

Estas diferencias son resultado del propio origen de los diferentes sectores y de las sucesivas operaciones de concentración de precariedad y realojos, que ha propiciado dinámicas de desplazamiento de la población interna y externa (Torres, 2005, pp. 27-34; Vorauer, 2011, pp. 112-116). Este proceso comienza con la construcción de los refugios o Casas Bajas prefabricadas en La Paz a finales de los 60. Continúa con la construcción en 1970 de los bloques cooperativistas de La Oliva de mejores condiciones, y los bloques de Las Letanías, donde se realojan algunos de los vecinos de Las Casas Bajas, dejando su lugar a población en peores condiciones. Después, en 1976, se construyen las viviendas de Murillo, sector hacia donde se desplaza parte de la pobla- 
ción de Las Letanías y los vecinos desalojados de las Casas Bajas. En los dos años siguientes se construyen los bloques de Antonio Machado y Martínez Montañés, que pasan a alojar a los últimos habitantes de las Casas Bajas tras su desmantelamiento final. En el espacio liberado se construyen en 1981 los bloques de Paz y Amistad, que reciben parte de la población que ha mejorado su situación de Las Letanías y Murillo. Finalmente, en los 90, se construyen los bloques cooperativistas alrededor de La Oliva (San Antonio, Giralda Sur, Nueva Europa, San Cristóbal, Cielo Azul, Sideromineros y Getsemaní), dando lugar a nuevos desplazamientos internos y también a la llegada de nuevos vecinos en condiciones menos vulnerables.

\section{La estrategia}

El Plan pretende dar respuesta a los fallos de las actuaciones públicas anteriores y a las demandas vecinales que

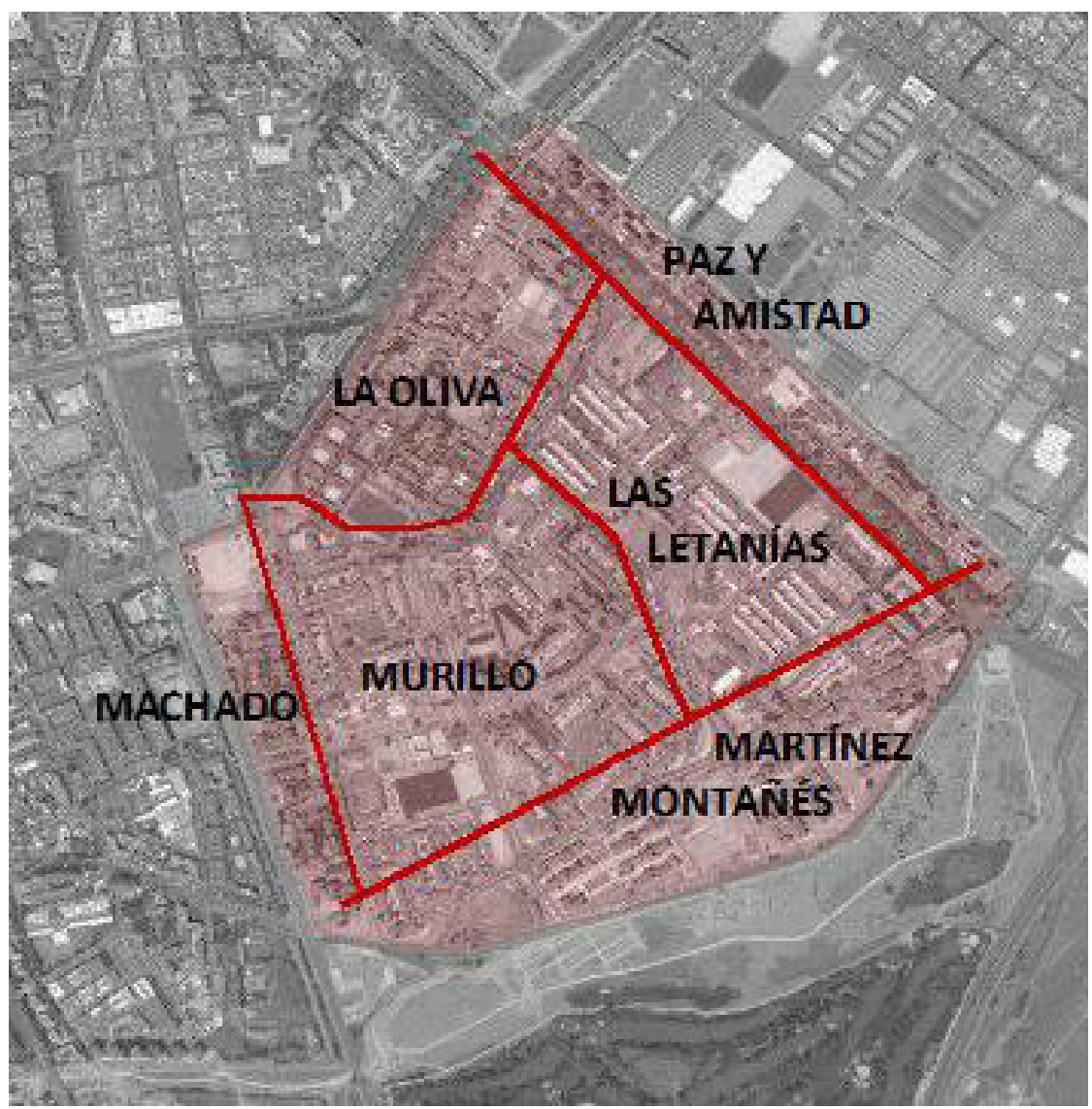

Figura 5. Delimitación del ámbito. lidades, una mayor implicación y capacidad de decisión de la base ciudadana organizada, y centrar la atención en los problemas de convivencia vecinal.

Se apuesta por la integralidad de temas, procedimientos y procesos en el análisis, planificación, intervención y gestión, para hacer frente a las distintas dimensiones de la marginalización. Esto parte de la idea de que el nivel de marginalización y los problemas de convivencia vecinal están relacionados con los problemas de habitabilidad y salubridad. Vorauer (2011) relaciona el objetivo segregacionista que se aprecia desde el origen de este polígono de vivienda social con la degeneración física y social que se materializa en un proceso de marginalización social involuntaria. Teniendo en cuenta la influencia del espacio macro sobre lo micro, considera que la rehabilitación integral es la estrategia más adecuada para revitalizar el área, pero puntualiza que ha de ir acompañada de un compromiso y apoyo de esta estrategia de los agentes a escala macro a largo plazo.

Por su lado, a la luz de los resultados en Almanjáyar (Granada), Torres (2005) también recomienda una intervención integral, que además de regularizar la titularidad de las viviendas, siga un proyecto de Pedagogía Urbana.

En la práctica, el objetivo de la integralidad lleva a crear una figura excepcional, el Comisionado del Polígono Sur, que pondrá en marcha un Plan Integral basado en la adaptación territorial de las políticas, programas y actuaciones, abordando aspectos esenciales como: participación ciudadana y mediación, urbanismo y convivencia, inserción sociolaboral y promoción de la iniciativa económica, salud comunitaria e intervención socioeducativa y familiar. Las acciones van encaminadas a mejorar los recorridos internos y las conexiones con otras partes de la ciudad y a potenciar los espacios y equipamientos sociales y educativos 
para trabajar sobre la convivencia vecinal, además de abordar problemas sociales básicos.

Este programa servirá de marco estratégico para alinear distintos Planes Sectoriales y proyectos de intervención complementarios, como el Plan URBAN Sur 2008-2013 o el Plan Especial de Mejora Urbana Martínez Montañés 2008. Además, busca complementar las actuaciones previstas en la zona en el PGOU 2006, como el soterramiento de la línea ferroviaria o el derribo del muro del polígono industrial de Hytasa, paralizadas hasta la fecha.

Esta forma de abordar la problemática de los barrios más desfavorecidos recibe una mención especial en los VI Premios Europeos de Urbanismo 2009.

\section{Resultados del análisis alternativo propuesto}

La evaluación para la validación de método se ha realizado en 2013, a partir de un fuerte trabajo de campo basado en recorridos de reconocimiento y entrevistas en profundidad y grupos de discusión con representantes de la diversidad de actores implicados, ${ }^{1}$ sobre el desarrollo del proceso hasta ese momento y las oportunidades existentes para el desarrollo de la zona.

En cuanto al funcionamiento y la capacidad de acción de la diversidad de actores, en este caso se apuesta por el trabajo sociocomunitario como estrategia de transformación social, y destacan como actores de enlace, el Comisionado, los equipos de zona de educación, formación y empleo, y el equipo encargado de las actuaciones de rehabilitación que cuenta con un perfil social y técnico.

Según el esquema de la Figura 6, en la evaluación realizada, destacan como dinámicas externas positivas la práctica acumulada de las políticas andaluzas de intervención integrada en barriadas, y como dinámicas internas positivas la experiencia previa de colaboración entre asociaciones, dispositivos y equipos de zona, que se ha ido consolidando en la zona. También destacan el capital social y los recursos movilizados en estas iniciativas de colaboración, gracias principalmente al trabajo de los equipos de zona, las asociaciones vecinales y las coordinadoras más activas. Resulta igualmente importante la función de los espacios relación como las plazoletas y el Centro Cívico El Esqueleto, así como las visiones compartidas entre los actores del barrio sobre la transformación de la zona.

En contra, se detectan como dinámicas externas negativas la falta de un entendimiento común entre áreas y niveles administrativos, y entre los cuerpos técnicos, directivos y políticos de los organismos responsables. A nivel interno, se identifica el rechazo entre barriadas y los problemas de convivencia vecinal en las barriadas más marginalizadas. Se detectan problemas de comunicación y coordinación por la escasa capacidad del Comisionado de comprometer a los organismos superiores responsables y de simplificar los procedimientos de colaboración formal. En cuanto a la articulación de actores y actuaciones, en este caso se detectan problemas por el uso conflictivo de algunas explanadas y espacios inter-bloque, la dificultad de construir y mantener relaciones de confianza con la población debido en gran medida a la respuesta ambigua de la administración y la rigidez del modelo de colaboración impuesto, y la falta de implicación de los organismos responsables superiores en la construcción de la visión estratégica y la adopción de compromisos. 

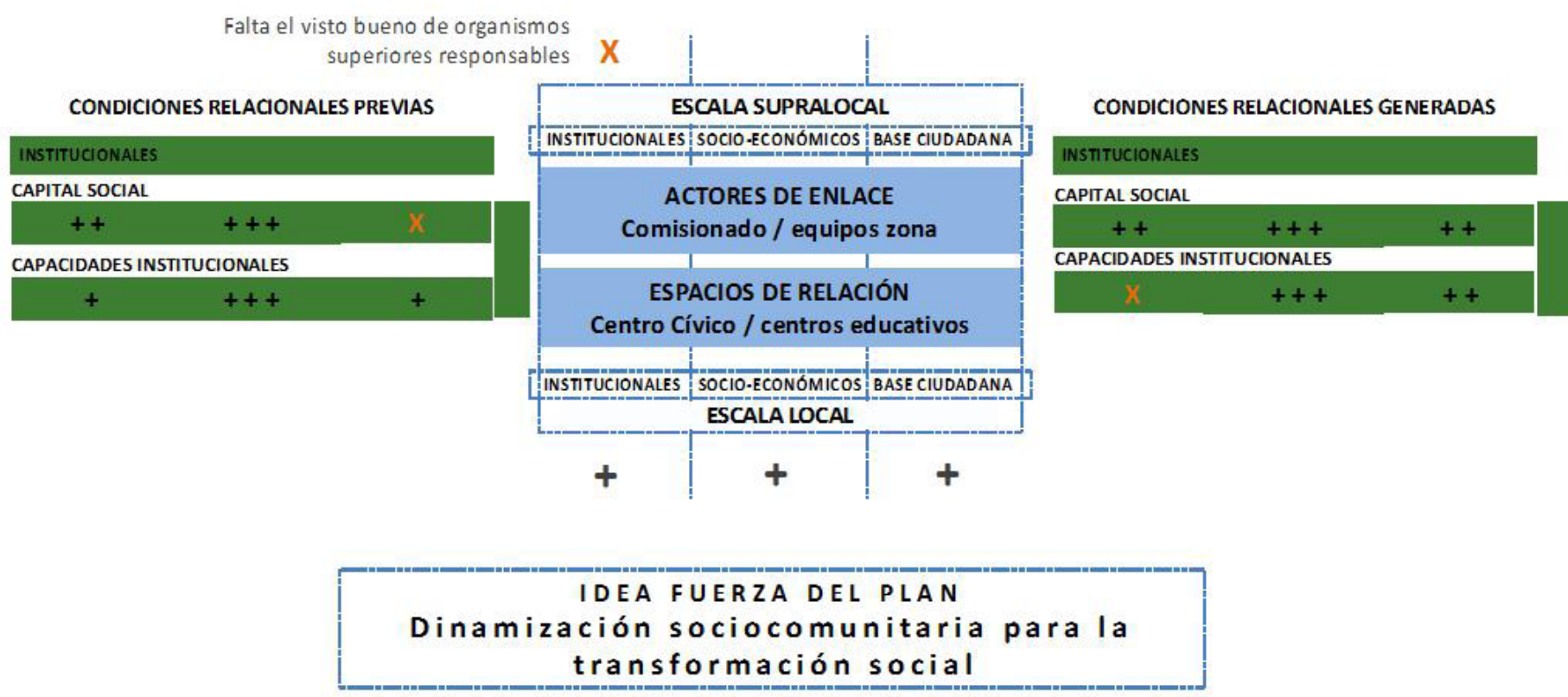

En el proceso de planificación se parte de una fuerte participación en el diagnóstico y la definición de propuestas para el Plan Integral, que acaba en un esquema más técnico ante los problemas para poner en marcha las actuaciones de manera participativa y en red teniendo en cuenta la rígida estructura administrativa por áreas y competencias.
Figura 6. Resultados del esquema del funcionamiento del sistema de acción. Identificación de los conjuntos o agentes de cambio, de los cambios en las condiciones relacionales y del apoyo a la idea fuerza del Plan. Elaboración propia.

\begin{tabular}{|c|c|c|c|c|c|c|c|c|c|c|c|c|c|c|c|c|c|c|c|c|c|c|c|c|}
\hline \multicolumn{5}{|c|}{$\begin{array}{l}\text { SITUACION DE } \\
\text { PARTIDA }\end{array}$} & \multicolumn{4}{|c|}{$\begin{array}{l}\text { ANAUSIS } \\
\text { ESTRATÉGICO }\end{array}$} & \multicolumn{4}{|c|}{$\begin{array}{l}\text { PROPUE STA } \\
\text { VABLE }\end{array}$} & \multicolumn{4}{|c|}{$\begin{array}{l}\text { ELABORACION } \\
\text { DELPLAN }\end{array}$} & \multicolumn{4}{|c|}{$\begin{array}{l}\text { ACCION } \\
\text { COLECTIVA }\end{array}$} & \multicolumn{4}{|c|}{$\begin{array}{l}\text { SITUACION } \\
\text { OBJE TIVO }\end{array}$} \\
\hline+ & + & + & + & + & + & + & + & + & - & + & - & - & + & + & + & - & - & - & - & + & 0 & + & + & + \\
\hline \multicolumn{25}{|c|}{ PROCE SO CONTINUO DE GE STIÓN INTE GRADA RELACIONAL } \\
\hline \multicolumn{5}{|c|}{ Criterios } & \multicolumn{7}{|c|}{ ACTORES } & & \multicolumn{6}{|c|}{ RECURSOS } & \multicolumn{6}{|c|}{ IN STRUMENTOS } \\
\hline \multicolumn{5}{|c|}{ MULTIDIMENS ЮNAL } & \multicolumn{7}{|c|}{ - } & & \multicolumn{6}{|c|}{+} & \multicolumn{6}{|c|}{+} \\
\hline \multicolumn{5}{|c|}{ MULT R GENTE } & \multicolumn{7}{|c|}{0} & & \multicolumn{6}{|c|}{-} & \multicolumn{6}{|c|}{ - } \\
\hline \multicolumn{5}{|c|}{ MULTIESCA LAR } & & & & $4=$ & & & & & \multicolumn{6}{|c|}{0} & \multicolumn{6}{|c|}{ - } \\
\hline
\end{tabular}

\section{Valoración de las implicaciones sociales según el método} de análisis propuesto

En cuanto a las implicaciones en el nivel de organización del tipo de participación que se ha dado en el proceso de planificación y gestión, se observa que en este caso las redes de colaboración social y socio-institucional se han visto reforzadas en el proceso, pero no precisamente por el trabajo del Comisionado, sino por el compromiso y la capacidad de trabajo en red demostrada por asociaciones y entidades del barrio que han puesto en marcha actividades sociocomunitarias que han mejorado las capacidades básicas de grupos vulnerables. En cambio, el trabajo realizado de formalización de comunidades de vecinos y de iniciativas de gestión comunitaria en las zonas comunes, soportales y espacios interbloque en el Plan de Rehabilitación ha tenido un impacto po-
Figura 7. Resultados del Diagrama de momentos del proceso de planificación modular y gestión relacional. Elaboración propia. 
sitivo, aunque al final han sido menos de las previstas. Sin embargo, la estructura técnica en la que se convierte el Comisionado va en contra de las relaciones de confianza que ha impulsado Surco en la gestión social del proyecto de Rehabilitación y también de las relaciones de confianza impulsadas en el trabajo socio-comunitario desarrollado por el equipo educativo de zona.

\section{Valoración según la percepción de la diversidad de actores $^{2}$}

En cuanto al nivel de integración (entrevista a representantes de la antigua EPSA, actual AVRA), el modelo no ha conseguido la integralidad y participación que pretendía. Se ha cambiado la imagen física y se ha invertido una gran cantidad de ayudas, pero sigue siendo una de las zonas más desfavorecidas.

Los representantes de las asociaciones vecinales tradicionales entrevistados acusan una permisividad institucional y un falta de autoridad en la zona, junto con una respuesta institucional descoordinada y en ocasiones contradictoria, que se percibe como una falta de voluntad institucional por cumplir con su responsabilidad y facilitar realmente la integración de las barriadas en el resto de la ciudad.

Además, los representantes de organizaciones empresariales como FEANSAL, advierten que este desinterés y la falta de información fuera de la zona sobre lo que se está haciendo en el Polígono Sur, hace que sea muy complicado desarrollar políticas que aborden estas diferencias territoriales.

En cuanto a la cohesión interna, en este caso se aprecia una diferencia entre barriadas por el régimen de tenencia y el nivel de rotación de la población. En las barriadas de vivienda en propiedad se ha producido un estancamiento que ha favorecido las relaciones entre los vecinos en un clima de normalidad, mientras que en las de vivienda social en alquiler se ha vivido una llegada continua de nuevos vecinos en situaciones críticas que dificulta generar lazos vecinales. Esta situación provoca un rechazo de los vecinos de las barriadas más normalizadas hacia las zonas más marginales. En este sentido, desde la base ciudadana se critica duramente el efecto negativo de los numerosos realojos programados sin ningún tipo de acompañamiento social. Surco, el equipo técnico encargado del proyecto de rehabilitación del parque de vivienda obsoleto, considera también desacertado el acuerdo alcanzado para la adjudicación de viviendas a favor de los vecinos empadronados en el Polígono Sur sin vivienda, ya que de esta forma no se favorece que vengan nuevos vecinos de otras zonas.

Frente a los problemas de convivencia vecinal, los representantes de la base ciudadana organizada entrevistados defienden el valor del importante trabajo de apoyo a las familias que ya se estaba desarrollando. Los responsables de los centros educativos y los programas socioeducativos entrevistados consideran que hace falta seguir trabajando por la educación en valores, la mejora de la autoestima y la mejora de la convivencia vecinal y familiar.

Por otro lado, se reconoce la repercusión de los proyectos sociales y educativos innovadores (los programas del Centro de Educación de Adultos, la Comunidad de Aprendizaje del Colegio Andalucía, el proyecto social de la Residencia universitaria Flora Tristán) y de nuevos equipamientos de rango ciudad (la Escuela de Empresas), a la hora

2 Entrevistas realizadas en noviembre de 2013. 
imagen negativa que difunden los medios y que los vecinos se sientan reconocidos.

Resulta especialmente interesante el trabajo sociocomunitario de estas entidades, como la Residencia Flora Tristán, que sigue una estrategia de intervención social en comunidades al estilo de la que se ha seguido en el Colegio Mayor Universitario La Coma, creado en 1994 en Paterna, Valencia, bajo las directrices del reconocido teórico de trabajo social, Joaquín García Roca.

Sin embargo, la entrevista realizada a un representante de la Comisión de empleo y desarrollo, advierte que el descontento que se vive en el barrio frente a las señales contradictorias que les lanza la administración, ha ensombrecido el trabajo que se ha hecho a favor de la integración como el Plan Educativo, la mejora de la red de equipamientos colectivos y de espacios públicos de relación, o los itinerarios completos y coordinados de formación y empleo.

La responsable entrevistada de la antigua EPSA reconoce que la viabilidad de las opciones de mejora del Polígono sería mayor si todos estuvieran convencidos de la importancia de alcanzar una mayor cooperación interadministrativa. En este sentido, en el caso de la regularización de viviendas y de la rehabilitación de bloques, EPSA se ha encontrado con que ha faltado un mayor apoyo de otros servicios públicos de zona para la gestión social de las actuaciones.

En cuanto al acceso a las oportunidades generadas, los representantes entrevistados de las asociaciones vecinales de las zonas más normalizadas se han sentido desplazados. Critican que los recursos y programas se hayan ajustado a las barriadas más marginales, y que al final, los vecinos de las zonas más normalizadas no tengan acceso a los nuevos recursos por desconocimiento o porque la oferta no se ajusta a sus necesidades. Además, también subrayan que el proceso para conseguir nuevos recursos y programas para el Polígono ha sido muy lento, y que las asociaciones y entidades del barrio han demostrado una capacidad de respuesta más rápida y cercana a los vecinos.

Desde la Comisión de empleo y desarrollo se reconoce que ha habido fallos de comunicación para difundir mejor dentro y fuera del barrio los resultados positivos, llegar a la población más vulnerable, y comunicar los resultados que no son tan visibles ni medibles como los resultados de procedimientos de trabajo basados en la participación y la colaboración.

Entre los entrevistados de los distintos servicios, dispositivos y equipos de zona no se aprecia un problema de falta de conocimiento de los recursos, sino de apropiación de los mismos que requiere tender relaciones de confianza. En este sentido, el Centro Cívico se ha revelado como un espacio más cercano a las entidades y a los vecinos que "los cascarones" donde se han ubicado el Comisionado y el Centro de Orientación y Dinamización para el Empleo (CODE).

En cuanto a los resultados de la operación de rehabilitación, desde el equipo Surco encargado del proyecto, son conscientes de que el apoyo institucional ha condicionado los trabajos paralelos con las familias, y al final, la repercusión en la convivencia vecinal ha sido inferior a la esperada, e incluso, en ocasiones, se ha vuelto a situaciones iniciales.

En definitiva, los entrevistados reconocen que no se ha conseguido mejorar la integración entre las barriadas, y que tampoco se ha integrado el barrio en la ciudad ni mucho menos en las decisiones políticas de desarrollo de la ciudad.

Y en cuanto a los problemas de accesibilidad efectiva a las oportuni- 
dades generadas, se advierte que se ha dado un problema de apropiación de los recursos porque se ha centrado la atención en la población de las zonas más marginales y en las demandas de los movimientos vecinales, que no reflejan el perfil completo de la población de todas las barriadas. Además, los problemas de financiación han provocado parones en las actuaciones o que los proyectos queden incompletos, y esto ha limitado el alcance esperado.

\section{Puntos críticos detectados en el análisis}

Esta lectura del Plan Integral del Polígono Sur muestra como principal punto débil la falta de autoridad de ejecución de la figura del Comisionado para exigir un compromiso firme y mantenido en el tiempo de las áreas municipales y consejerías afectadas. Esto pone en cuestión su validez en los últimos años en los que no se cuenta con tantos recursos públicos y en los que además las asociaciones y entidades entran en competencia para conseguir ayudas públicas que garanticen la continuidad de sus programas. Ahora no se trata de coordinar recursos en el territorio, sino de alinear los intereses, recursos y programas de cada uno para maximizar el impacto de las actuaciones. Pero resulta difícil atraer el interés de los servicios públicos si no ven que se van a beneficiar de ayudas públicas dirigidas a esta zona.

Otra dificultad añadida ha sido abordar las diferencias entre las distintas barriadas, con diferentes niveles de marginalidad y de organización de la base ciudadana, que exige utilizar medidas diferentes pero bajo los criterios y objetivos comunes del Plan Integral.

Además, del análisis de las entrevistas, se detectan los siguientes retos:

- En el ámbito institucional, los responsables presentes en la zona reconocen que la figura híbrida del Comisionado ha resultado una estructura poco flexible sin un reparto claro de responsabilidades ni capacidad de acción que ha complicado y retrasado la intervención y ha provocado conflictos internos.

Apuntan que la aplicación del modelo de actuación, basado en la integralidad y la participación, ha fallado por dos motivos principales. Primero, por la falta de un entendimiento común sobre el tipo de intervención que necesitaba la zona, y segundo, por la ambigüedad mostrada por las administraciones superiores responsables que en la práctica no están a favor de descentralizar la acción ni de facilitar el reparto de responsabilidades para ello.

El esquema de descentralización y la capacidad de organización demostrada por los técnicos de zona han puesto en cuestión la forma tradicional de trabajar de la administración. Esto evidencia la necesidad de innovar en la gestión pública para adaptarse a las demandas actuales, aprovechar los recursos disponibles y contemplar fórmulas innovadoras de financiación y gestión.

Frente a la insatisfacción generalizada con el alcance de los resultados, señalan que sería conveniente promover actuaciones participativas y colaborativas menos ambiciosas, e ir poco a poco engranando a los diferentes actores y validando la acción a lo largo del proceso, construyendo así con la práctica un entendimiento común y las capacidades necesarias para poner en marcha modelos más complejos.

En definitiva, consideran que se necesita seguir trabajando en 
la línea de la integralidad pero que en la nueva etapa, el Comisionado debería resolver las ambigüedades y conflictos que ha causado la anterior, y establecer una estructura más sencilla y operativa.

- Los servicios en el barrio y los equipos de zona también coinciden en señalar que la falta de claridad en el reparto de competencias ha intensificado las diferencias internas, y que no siempre los que han colaborado más con el Comisionado han sido los más representativos.

En general, critican que la falta de autoridad lo que ha hecho en realidad es duplicar los filtros por los que tienen que pasar las iniciativas que surgen desde el barrio, y esto ha añadido más burocracia y complejidad. Y a su vez, como no ha integrado iniciativas locales en marcha, se han dado duplicidades entre las acciones del Plan Integral y las que ya venían desarrollando entidades, equipos de zona y asociaciones vecinales. También reprochan al Comisionado que haya intentado coordinar servicios en red en base a la voluntad de cada agente implicado y no de un compromiso político real. En este sentido, los técnicos de zona demandan un mayor respaldo institucional a su trabajo en el territorio y también contar con un equipo experto en gestión y mediación para resolver las tensiones que puedan surgir ente dispositivos de las barriadas, con la intención de favorecer el trabajo en red en vez de la competencia entre entidades que genera la concurrencia competitiva por las subvenciones públicas.

En este proceso, la función que ha ejercido Centro Cívico El Esqueleto como punto de interacción entre vecinos, asociaciones y entidades, evidencia la importancia de crear espacios de participación en los que se puedan poner en marcha iniciativas desde el barrio pero sin filtros administrativos ni presiones políticas o económicas.

- Por su parte, los expertos que han participado advierten también las limitaciones del Comisionado al tratarse de una figura intermedia con el papel de gestor pero sin recursos. Observan que la práctica ha demostrado que no resulta efectivo imponer un método de intervención y gestión que no se ajuste al contexto. En este caso ha faltado un trabajo previo para generar conciencia de trabajo en red, fijar un reparto de responsabilidades y compromisos, y dejar bien claro lo que se puede esperar del proceso participativo.

Algunos expertos ponen en cuestión el carácter de excepcionalidad y señalan que sería más interesante que los servicios y programas públicos en la zona fueran igual de ambiciosos que en otras partes de la ciudad, no subestimar las capacidades de la población, sino desarrollarlas de forma constructiva para poder iniciar el cambio.

En el caso concreto de las actuaciones de rehabilitación de los edificios, lo más complicado ha sido motivar e implicar a los vecinos en un ambiente en el que rechazan y sospechan de la administración. Esta postura ha mejorado ante los resultados de los primeros bloques.

- Igualmente, la base ciudadana organizada critica la falta de autoridad política y de ejecución del Comisionado, la falta de apoyo a las iniciativas locales y el alcance limitado de los resultados a pesar del esfuerzo y apoyo económico que se concentraron en 
un determinado momento en la zona. Consideran que ha faltado contar más con el trabajo que ya se estaba haciendo en la zona y conceder mayor capacidad de acción a los agentes del barrio más comprometidos con la transformación social. Como resultado se ha intensificado la desconfianza hacia las instituciones y esto dificulta mantener vivo el interés de los vecinos de colaborar en el trabajo del Comisionado.

Además, a diferencia de lo que se ha hecho hasta ahora, entienden que las formas de participación e intervención se tendrán que ajustar a la población de las diferentes barriadas. Así, en el caso de las más normalizadas que han vivido una rotación de población menor, habrá que tener en cuenta a la población actual envejecida con una larga trayectoria reivindicativa. Mientras que en las barriadas más marginales, sería conveniente trabajar las capacidades sociales y culturales básicas que faciliten la participación de la población conflictiva.

En general, se observa un interés general por intervenir en el Polígono en base a criterios de integralidad, participación y adaptación al territorio tal y como se proponía desde el Comisionado. Aunque se reconoce que se han hecho mejoras en el barrio, se percibe un descontento generalizado con los resultados alcanzados y la validez de la estructura del Comisionado, que no ha conseguido generar un entendimiento común ni contar con el respaldo político necesario.

De cara a dar continuidad a las dinámicas iniciadas, sería fundamental resolver los problemas de reparto de poder, competencias y responsabilidades, integrar las iniciativas de los equipos de zona y el resto de agentes del barrio para evitar duplicidades, simplificar los procedimientos de comunicación, toma de decisiones y fiscalización de las ayudas públicas, un mayor compromiso y apoyo de las administraciones superiores de las que dependen los servicios de zona, y establecer espacios para impulsar y consolidar iniciativas desde el barrio.

\section{Conclusiones finales}

A la luz de los resultados de la evaluación del desarrollo del Plan Integral del Polígono Sur, basada en una mirada sistémica e integrada, se detecta la importancia de:

- Responder a una demanda real de la población.

- Construir un entendimiento común entre la diversidad de áreas y niveles administrativos con competencias en los distintos campos de acción que se comprometan de manera firme a actuar dentro del marco estratégico a definir que ha de ser consecuente y estar articulado con la estrategia-ciudad de regeneración urbana.

- Contar con equipos de gestión con autonomía suficiente y capacidad de acción.

- Contar con equipos multidisciplinares para la redacción del Plan y la dinamización del mismo con apoyo de expertos en participación y mediación de conflictos.

- Acordar entre la diversidad de actores las reglas de comunicación, toma de decisiones y de adopción y seguimiento de compromisos. 
- Desarrollar campañas para sensibilizar y generar demanda sobre intervenciones integradas y participativas y la rehabilitación integral frente a actuaciones dispersas.

- Disponer de medios y tiempo para realizar un trabajo previo dirigido a identificar y reforzar los elementos dinamizadores y articuladores y mejorar las condiciones previas de interacción para tratar con la población y los responsables institucionales.

En definitiva, se evidencia que sería deseable que los programas de ayudas a la regeneración de barrios desfavorecidos apoyen también este trabajo previo de sensibilización, movilización, posicionamiento y organización entre la diversidad de actores de distintos sectores y escalas.

\section{Relevancia de los resultados en el futuro}

Este ejercicio de análisis ha puesto de manifiesto el valor de métodos e instrumentos de análisis de tipo sistémico e integrado a la hora de visualizar factores que influyen en las opciones de cambio que de otra manera serían difíciles de evidenciar, como las condiciones de relación y organización.

En este caso se ha utilizado este método en el momento de cambio en la dirección del Comisionado en todos los sentidos, lo que acaba arrojando una imagen de un proceso cambiante en un momento concreto. Esto ha servido para reconocer el peso de las condiciones del funcionamiento del sistema de acción y del diagrama del proceso de planificación en todas las fases del proceso, pero sería deseable utilizar métodos e instrumentos de este tipo no solo como herramientas de evaluación, sino también como herramientas para acompañar el diseño y desarrollo de procesos de tipo participativo, y poner la atención a los momentos de inflexión del mismo, como el derivado de este momento de incertidumbre. 


\section{Referencias bibliográficas}

\section{Marco teórico, conceptual y metodológico}

Blanco, I., Bonet, J., Del Valle, G. y Sánchez, J. (2006). La dimensión relacional de la exclusión social y las políticas de protección social. Barcelona: Universitat Autònoma de Barcelona.

Blanco, I., Bonet, J. y Walliser, A. (2011). Urban governance and regenerations policies in historic city centers: Madrid and Barcelona. Urban Research $\mathcal{E}$ Practice, 4 (3), 326-343.

Bonet, J. (2006). La vulnerabilidad relacional: análisis del fenómeno y pautas de intervención. REDES, Monográfico: participación y análisis de redes de redes, $11(4)$

Borja, J. (2003). La ciudad conquistada. Madrid: Alianza Editorial.

Castel, R. (1995). De la exclusión como estado a la vulnerabilidad como proceso. Arquipiélago: Cuadernos de crítica de la cultura, 21, 27-36.

Castells, M. (2006). La sociedad red. Madrid: Alianza Editorial.

Castrillo, M., Matesanz, Á., Sánchez, D. y Sevilla, A. (2014). ¿Regeneración urbana? deconstrucción y reconstrucción de un concepto incuestionado. $P a$ peles de relaciones ecosociales y cambio global, 126, 129139.

Centelles, J. (2006). El buen gobierno de la ciudad. Estrategias urbanas y política relacional. La Paz: Plural Editores.

De Manuel, E. (2008). Participar para Re-cuperar la Polis. En Encina, J. et al. (coords.), La ciudad a escala humana. Sevilla: Atrapasueños, UNILCO y Universidad y Compromiso Social, pp. 89-102.

Fernández, V. y Dundas, M.V. (2008). Innovación, territorio y aglomeración: discutiendo sus vínculos y limitaciones desde una perspectiva multiescalar y multidimensional del desarrollo. REDES, 14 (17), 191-218.

Forrest, R. y Kearns, A. (2001). Social cohesion, social capital and the neighborhood. Urban Studies, 38 (12), 2125-2143.

Gittel, R. y Vidal, A. (1998). Community organizing. Building social capital as a development strategy. SAGE. DOI: http://dx.doi.org/10.4135/9781452220567.

González, S. y Vigar, G. (2008). Community influence and the contemporary local state. Potentials and contradictions in the neoliberal city. City, 12 (1), 64-78.

Gottweis, H. (2003). Theoretical strategies of post-structuralist policy analysis: towards an analytics of government. En Hajer, M. y Wagenaar, H. (eds.), Deliberative Policy Analysis Hardback: Under- standing Governance in the Network Society. New York: Cambridge University Press, pp. 247-265.

Gualini, E. (2006). The rescaling of governance in Europe: new spatial and institutional rationales. European Planning Studies, 14 (7), 881-904.

Hajer, M. y Wagenaar, H. (2003). Introduction. En Hajer, M. y Wagenaar, H. (eds.), Deliberative Policy Analysis Hardback: Understanding Governance in the Network Society. New York: Cambridge University Press, pp. 3-32.

Harvey, D. (2008). The right to the city. New Left Review, 53, 23-40.

Healey, P. (1997). Collaborative planning: shaping places in fragmented societies. London: MacMillan.

Healey, P. (1998). Building institutional capacity through collaborative approaches to urban planning. Environment and Planning, 3, 1531-1546.

Healey, P. (2003). Collaborative planning in perspective. Planning Theory, 2 (2), 101-123.

Hernández, A. (1997). Análisis urbanístico de barrios vulnerables. Catálogo de áreas vulnerables española. Cuadernos de investigación urbanística, 19.

Hernández, A., Matesanz, Á. y García, C. (2016) . Atlas de barrios vulnerables de España. 12 Ciudades 1991/2001/2006. Madrid: Instituto Juan de Herrera.

Innes. J.E. y Booher, D. (2003). Collaborative policymaking: governance through dialogue. En Hajer, M. y Wagenaar, H. (eds.), Deliberative Policy Analysis Hardback: Understanding Governance in the Network Society. New York: Cambridge University Press, pp. 33-60.

Jacobs, J. (2011). Muerte y vida de las grandes ciudades. (Trad. Á. Abad y A. Useros). Madrid: Capitan Swing. (Original en inglés, 1961).

Marcuse, P. (2012). Whose right(s) to what city? En Brenner, N., Marcuse P. y Mayer M. (eds.), Cities for people not for profit: critical urban theory and the right to the city, Routledge, pp. 24-42.

Mayer, M. (2012) The right to the city in urban social movements. En Brenner, N., Marcuse P. y Mayer M. (eds.), Cities for people not for profit: critical urban theory and the right to the city, Routledge, pp. 63-85.

Montañés, M. (2009). Metodología y técnica participativa: teoría y práctica de una estrategia de investigación. Barcelona: Universitat Oberta de Catalunya.

Morin, E. (1990). La introducción al pensamiento complejo. Barcelona: Gedisa.

Moulaert, F. (2000). Globalization and integrated area development in European cities. Oxford: Oxford University Press.

Nel.lo, O. (2008). Contra la segregación urbana y por la cohesión social: La Ley de Barrios de Cataluña. En Ciudades en (re)construcción: necesidades sociales, 
transformación y mejora de barrios. Barcelona: Diputació de Barcelona - Xarxa de municipis, pp. 233-254.

OCDE (1998) Integrating distressed urban areas. Paris: OCDE Publishing. DOI: 10.1787/9789264162884-en

Ortiz, E. (2010). Derecho a la ciudad, producción social y gestión participativa del hábitat. La promoción de iniciativas comunitarias incluyentes en la Ciudad de México. Hábitat y Sociedad, 1, 55-70.

Pelli, V.S. (2010). La gestión de la producción social del hábitat. Hábitat y Sociedad, 1, 39-54.

Rubio, J. (2011, abril). Rehabilitación urbana en Zaragoza: la experiencia reciente. Trabajo presentado en las Jornadas: regeneración urbana integral. Recuperado el 10 de diciembre de 2015, de: www. five.es/descargas/archivos/Regenera/04_Juan_Rubio.pdf.

Salet, W. y Majoor, S. (2008). The enlargement of local power in trans-scalar strategies of planning: recent tendencies in two European cases. Geojournal, 72 (1), 91-103.

Schnur, O. (2005) Exploring social capital as an urban neighbourhood resource: empirical findings and strategic conclusions of a case study in Berlin Moabit. Tijdschrift voor economische en sociale geografie, 96 (5), 488-505.

Sen, A. (1992). Inequality Re-Examined. Oxford: Oxford University Press.

Sen, A. (2005). Human rights and capabilities. Journal of Human Development, 6 (2), 151-166.

Sevilla, Á. (2010). Urbanismo, biopolítica, gubernamentalidad: vida y espacio en la renovación de los estudios urbanos. Boletín $C F+S$, 44, 41-49.

Skidmore, P., Bound, K. y Lownsbrough, H. (2006). Do policies to promote community participation in governance build social capital? York: Joseph Rowntree Foundation.

Subirats, J. (2004). Pobreza y exclusión social. Un análisis de la realidad española y europea. Estudios Sociales, 16.

Sungranyes, A. (2010). El derecho a la ciudad. Praxis de la utopía. Hábitat y Sociedad, 1, 71-79.

\section{Caso de estudio}

Carmona, A. (1999). Nosotras: Historias de mujeres del Poligono Sur de Sevilla. Sevilla: Diputación de Sevilla.

Carmona, J. (2008). Hacia el Polígono Sur 'imposible' que queremos. Algunas reflexiones sobre los procesos de desarrollo en la transformación del barrio. Cuchará y paso atrá, 19, 7-25.

Díaz, I. (2005). Un caso de segregación. El Polígono Sur. Sevilla: Departamento de geografía humana, Universidad de Sevilla.
Encina, J. y Zaragoza, J.M. (2008). A vueltas con el ilusionismo social. Cuchará y paso atrá, 18.

García Roca, J. y Mondaza, G. (2002). Jóvenes, universidad y compromiso social. Madrid: Narcea.

Gómez, D. (2011) Rehabilitación del Polígono Sur de Sevilla. Coordinación interadministrativa en los Planes Concertados de Rehabilitación de Viviendas, I Parte. Sevilla: EPSA.

Hernández, A. (2011). Análisis urbanístico de barrios vulnerables. Madrid: Instituto Juan de Herrera.

Laboratorio Urbano. (2010). El Plan Integral del Polígono Sur como modelo de actuación, La Ciudad Viva, 4. Recuperado el 10 de diciembre de 2015, de: www.laciudadviva.org/blogs / ?p=2497

López, J.M. (2012). El diseño participativo en programas de rehabilitación de vivienda. Tesis doctoral, Escuela Técnica Superior de Arquitectura, Universidad de Sevilla, Sevilla (inédita).

Maeztu, J. (2008, diciembre). Cohesión Social y Territorial: El Plan Integral del Polígono Sur de Sevilla. Trabajo presentado en la VI Conferencia AERYC, Cohesión social y gobernanza democrática para ciudades y regiones más inclusivas. Recuperado el 10 de diciembre de 2015, de: http:/ / myslide.es/documents / cohesion-social-y-territorial-el-plan-integraldel-poligono-sur-de-sevilla-de-jesus-maetzu.html.

Pascual, J.M. y Tarragona, M. (2009). Estrategia Territorial y Gobierno Relacional. Manual para la Planificación Estratégica de 2. ${ }^{a}$ Generación. Estrategias de calidad urbana. Sevilla: Junta de Andalucía.

Torres, F.J. (2013). Segregación urbana y exclusión social en Sevilla: el paradigma del Polígono Sur. Sevilla: Universidad de Sevilla, Fundación Focus Abengoa.

Torres, F.J. (2005). El análisis territorial aplicado al estudio de zonas urbanas marginadas. El caso del Polígono Sur en Sevilla. Sevilla: Junta de Andalucía.

Valenciano, D. y Collado, M. (2009). Cuando la palabra de las mujeres rompe el silencio. Historias de vida narradas por mujeres del Taller de Empleo Intervención Comunitaria Polígono Sur. Sevilla: Ayuntamiento de Sevilla. Recuperado el 10 de diciembre de 2015, de: www.poligonosursevilla.es/opencms/export/sites / epsur/recursos/documentos/Empleo_y_Formacion/CUANDO_LA_PALABRA_DE_LAS_MUJERES_ROMPE_EL_SILENCIO.pdf.

Vázquez, C. (2011). Diagnóstico de territorios desfavorecidos en la ciudad de Sevilla. Tres Barrios: Amate, Sur, Torreblanca, Norte. Sevilla: Delegación de Economía y Empleo, REDES Sevilla.

Vorauer, M. (2011). 'Las 3000': segregación social en el espacio urbano. Sevilla: Universidad de Sevilla. 
GALLEGO GAMAZO, Cristina. Análisis de la implicación de la diversidad de actores en el Plan Integral del Polígono Sur: una mirada sistémica e integrada. Hábitat y Sociedad, 2016, n. ${ }^{\circ}$ 9, pp. 55-74.

$<$ www.habitatysociedad.us.es>

http://dx.doi.org/10.12795/HabitatySociedad.2016.i9.04 